\title{
A rigorous measure of genome-wide genetic shuffling that takes into account crossover positions and Mendel's second law
}

\author{
Carl Veller ${ }^{\mathrm{a}, \mathrm{b}}$, Nancy Kleckner, ${ }^{\mathrm{c}, 1}$, and Martin A. Nowak ${ }^{\mathrm{a}, \mathrm{b}, \mathrm{d}}$

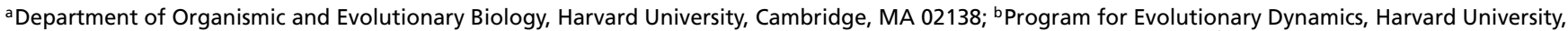 \\ Cambridge, MA 02138; 'Department of Molecular and Cellular Biology, Harvard University, Cambridge, MA 02138; and ${ }^{\mathrm{d} D e p a r t m e n t}$ of Mathematics, \\ Harvard University, Cambridge, MA 02138
}

Contributed by Nancy Kleckner, November 27, 2018 (sent for review October 11, 2018; reviewed by Molly Przeworski and Nadia Singh)

Comparative studies in evolutionary genetics rely critically on evaluation of the total amount of genetic shuffling that occurs during gamete production. Such studies have been hampered by the absence of a direct measure of this quantity. Existing measures consider crossing-over by simply counting the average number of crossovers per meiosis. This is qualitatively inadequate, because the positions of crossovers along a chromosome are also critical: a crossover toward the middle of a chromosome causes more shuffling than a crossover toward the tip. Moreover, traditional measures fail to consider shuffling from independent assortment of homologous chromosomes (Mendel's second law). Here, we present a rigorous measure of genome-wide shuffling that does not suffer from these limitations. We define the parameter $\bar{r}$ as the probability that the alleles at two randomly chosen loci are shuffled during gamete production. This measure can be decomposed into separate contributions from crossover number and position and from independent assortment. Intrinsic implications of this metric include the fact that $\bar{r}$ is larger when crossovers are more evenly spaced, which suggests a selective advantage of crossover interference. Utilization of $\bar{r}$ is enabled by powerful emergent methods for determining crossover positions either cytologically or by DNA sequencing. Application of our analysis to such data from human male and female reveals that $(i) \bar{r}$ in humans is close to its maximum possible value of $1 / 2$ and that (ii) this high level of shuffling is due almost entirely to independent assortment, the contribution of which is $\sim 30$ times greater than that of crossovers.

recombination | crossing-over | sex | meiosis

T he shuffling of maternally and paternally inherited genes during gamete production is an important process in sexual populations. It leads to genetic diversity among gametes and offspring (1), and thus aids adaptation to novel or changing environments (2). In addition, it improves the long-term efficiency of adaptation by allowing natural selection to act separately on distinct mutations (3-8). Genetic shuffling has further been implicated in protecting populations from rapidly evolving parasites $(9,10)$ and from the harmful invasion of selfish genetic complexes (11-14). The total amount of shuffling that occurs in gamete production is, therefore, a quantity of considerable importance and has been the subject of much empirical interest.

Correspondingly, comparative studies $(1,15-21)$ of genomewide shuffling have been carried out across species (reviewed in refs. 22-24), with implications ranging from distinguishing the evolutionary advantages of sex $(1,15,23-25)$ to testing the genomic effects of domestication $(15,17)$. A large literature has also studied male/female differences in shuffling $(14,26,27)$, prompting several evolutionary theories to explain these differences $(13,14,26-29)$, which could concomitantly be informative of the adaptive value of shuffling (26). There can also exist differences in shuffling across individuals of the same sex [e.g., in flies (30-34), mice (35-37), humans (37-42), and Arabidopsis (43); reviewed in ref. 44] and across gametes produced by the same individual $(37,39,45-49)$. Finally, comparisons have been made of the levels of shuffling within different chromosomes (50-52), with implications for which chromosomes are most susceptible to harboring selfish genetic complexes (13) or to be used as new sex chromosomes $(26,53)$.

Quantitative comparisons like these require a proper measure of genome-wide shuffling. Shuffling is caused both by crossovers (COs) and by independent assortment of homologous chromosomes (IA), ${ }^{*}$ which comprise "intrachromosomal" and "interchromosomal" shuffling, respectively. ${ }^{\dagger}$ In previous studies, the most widely used measures of shuffling have considered only the contribution of crossing-over and more specifically, $\mathrm{CO}$ frequency or map length. CO frequency is simply the number of COs that occur during meiotic prophase as measured either cytologically or from sequence data. Map length is average $\mathrm{CO}$ frequency multiplied by $50 \mathrm{cM}, 1 \mathrm{cM}$ being the map distance between two linked loci that are shuffled in $1 \%$ of gametes (54). Another measure that is sometimes used is the number of COs in excess of the haploid chromosome number (15). Since each bivalent usually requires at least one $\mathrm{CO}$ for its chromosomes to segregate properly (55), the "excess CO frequency" is the number of COs that contribute to shuffling beyond this supposed structurally required minimum. None of the above measures take into account shuffling caused by IA. A fourth measure of

\section{Significance \\ An important process in sexual organisms is the shuffling of maternal and paternal DNA in gametes by both crossing over and independent assortment of homologous chromosomes. Traditional measures of the total amount of genetic shuffling simply count the average number of crossovers, failing to take into account independent assortment and, importantly, the positions of crossovers. We develop a measure of total shuf- fling that takes into account these features: $\bar{r}$, the probability that a randomly chosen pair of loci shuffles their alleles in a gamete. Measuring $\bar{r}$ in humans, we find that total shuffling is close to its maximum possible value of one-half and that this high value is largely due to independent assortment.}

Author contributions: C.V. designed research; C.V. performed research; C.V., N.K., and M.A.N. analyzed data; and C.V., N.K., and M.A.N. wrote the paper.

Reviewers: M.P., Columbia University; and N.S., University of Oregon.

The authors declare no conflict of interest.

Published under the PNAS license.

Data deposition: MATLAB code for all calculations is available on GitHub (https:// github.com/cveller/rbar).

${ }^{1}$ To whom correspondence should be addressed. Email: kleckner@fas.harvard.edu.

This article contains supporting information online at www.pnas.org/lookup/suppl/doi:10. 1073/pnas.1817482116/-/DCSupplemental.

Published online January 11, 2019

* Gene conversion also causes shuffling but makes a negligible contribution as discussed below.

"Shuffling caused by either crossing-over or IA is referred to as "recombination" in the population genetics literature, but recombination has a different meaning-the breakage and rejoining of DNA molecules-in molecular biology. To avoid confusion, we use the unambiguous term "genetic shuffling" (or just "shuffling") throughout. 
aggregate shuffling, which does take into account IA, is Darlington's "recombination index" (RI) $(1,56,57)$, defined as the sum of the haploid chromosome number and $\mathrm{CO}$ frequency. The rationale for this measure derives from the fact that, given no chromatid interference in meiosis $(54,58)$, two linked loci separated by one or more COs shuffle their alleles with probability $1 / 2$ in the formation of a gamete, as if the loci were on separate chromosomes. The RI is, therefore, the average number of "freely recombining" segments per meiosis.

Importantly, none of these existing measures take into account the specific positions of COs on the chromosomes. Intuitively, however, $\mathrm{CO}$ position is a critical parameter. For example, a CO at the far tip of a pair of homologous chromosomes does little work in shuffling the genetic material of those chromosomes, while a $\mathrm{CO}$ in the middle causes much shuffling (Fig. 1). Additionally, two COs close together may cancel each other's effect (except for the few loci that are between them), and thus result in less allelic shuffling than two COs spaced farther apart.

Therefore, existing measures do not actually define the total genome-wide amount of shuffling, instead serving only as proxies for this critical parameter. This is not a trivial concern. There is significant heterogeneity in the chromosomal positioning of COs at all levels of comparison-between species $(16,59,60)$, populations within species (61-65), the sexes (14, 26, 66-69), individuals $(42,70-73)$, and different chromosomes $(50,69,74$, 75), all of which will impact the level of shuffling that these COs cause. Moreover, there can be major differences in chromosome number and size across species $(1,76,77)$, which will seriously influence the total amount of shuffling due to IA.

The fact that the positions of COs matter for the total amount of shuffling has been recognized for many years $(1,66,76,78-80)$. The need for a measure of total shuffling that accounts for $\mathrm{CO}$ positions has also previously been recognized. Indeed, Burt et al. (27) give an explicit formula, identical to Eq. 3 below, for "the proportion of the genome which recombines" (in the population genetics sense $^{\dagger}$ ). Colombo (81) also gives an explicit characterization of what we call $\bar{r}$ phrased as a generalization of the RI. Finally, Haag et al. (82), after noting that terminal COs cause little shuffling and that map length is, therefore, an imperfect measure of genome-wide shuffling, suggest a better measure to be "the average likelihood that a $\mathrm{CO}$ occurs between two randomly chosen genes." However, no mathematical expression that
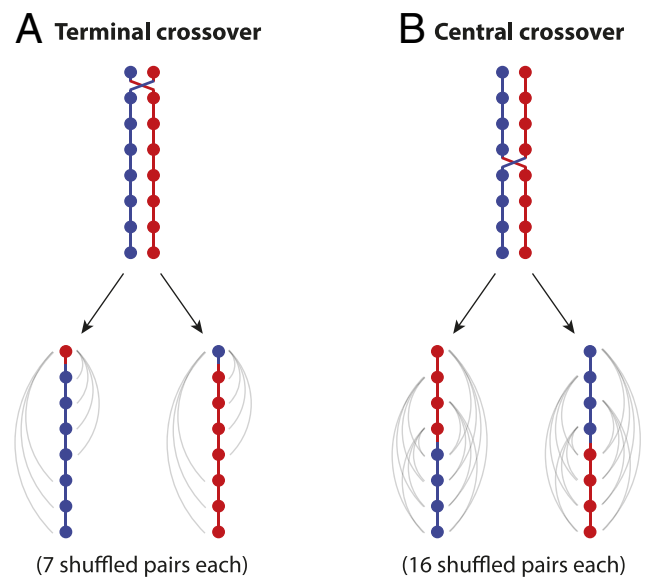

Fig. 1. The position of a $\mathrm{CO}$ affects the amount of genetic shuffling that it causes. The figure shows the number of shuffled locus pairs that result from crossing-over between two chromatids in a one-step meiosis. The chromosome is arbitrarily divided into eight loci. (A) A CO at the tip of the chromosome between the seventh and eighth loci causes 7 of 28 locus pairs to be shuffled in each resulting gamete. $(B)$ A CO in the middle of the chromosome between the fourth and fifth loci causes 16 of 28 locus pairs to be shuffled in each resulting gamete. The central $\mathrm{CO}$ thus causes more shuffling than the terminal co. incorporates $\mathrm{CO}$ position in the measurement of genome-wide shuffling has been developed and implemented.

Here, we present a simple intuitive measure of the genomewide level of shuffling. We define $\bar{r}$ as the probability that a randomly chosen pair of loci shuffles their alleles in meiosis, taking into account $\mathrm{CO}$ number and position as well as the contribution of IA. We have chosen the name " $\bar{r}$ " to echo classic population genetics terminology, where the parameter $r$ for a given pair of loci is the probability that they shuffle their alleles in a gamete. Our parameter $\bar{r}$ is simply this quantity averaged across all locus pairs.

$\bar{r}$ is the most direct metric of the primary effect of genetic shuffling, the diversification of gametes, and thus the transmission of new genetic combinations to offspring (1). $\bar{r}$ should be used when such diversification is the quantity of interest: for example, in judging the ability of a population to respond to a sudden change (or continual fluctuations) in its environment (2). Genetic shuffling also has indirect effects, such as the gradual reduction of allelic correlations (linkage disequilibrium) within populations over time $(6,7)$ and the prevention of invasion of selfish genetic complexes (11). Here, CO positions and IA again matter, so standard metrics that simply count COs remain inadequate. Therefore, $\bar{r}$ is likely a better measure in these cases as well, although we suggest (Discussion) modifications of $\bar{r}$ that more directly measure these quantities of interest.

In this work, we define the quantity $\bar{r}$, develop it mathematically and statistically, and document its intrinsic implications. We show how it can be decomposed into separate components deriving from COs and from IA. We then discuss the approaches now available to allow chromosome-specific and/or genome-wide measurement of $\mathrm{CO}$ positions. With these developments in hand, we present an application of $\bar{r}$ to quantitative evaluation of shuffling in human males and females.

\section{Derivation of $\bar{r}$}

$\bar{r}$ is the probability that the alleles at two randomly chosen loci are shuffled in the production of a gamete. It can be calculated with knowledge of $\mathrm{CO}$ positions on each chromosome in conjunction with knowledge of the fraction of the total genomic length (in base pairs) accounted for by each chromosome (chromosome lengths influence shuffling caused by both IA and COs). In what follows, we always assume that there are sufficiently many loci so that the difference between sampling them with or without replacement is negligible.

Formulas for $\bar{r}$. In the ideal situation, CO positions are defined for individual gametes, and the parental origins of the chromosome segments delimited by these COs are known. In this case, the proportion $p$ of the gamete's genome that is paternal can be determined, and the probability that the alleles of a randomly chosen pair of loci were shuffled during formation of the gamete (equivalently, the proportion of locus pairs at which the alleles are shuffled), either by crossing-over or by IA, is simply the product of the probabilities that one locus is of paternal origin (probability $p$ ) and the other is of maternal origin (probability $1-p$ ) in each of two possible combinations:

$$
\bar{r}=2 p(1-p)
$$

Such data can emerge directly from sequencing of single gametes (Fig. $2 B$ ) or of a diploid offspring in which the haploid contribution from a single gamete can be identified (Fig. 2C).

In some analyses, while CO positions for a gamete are known, the specific parental identities of the chromosome segments that these COs delimit are not known, because the gamete producer has been sequenced but its parents have not. In this case, we can nonetheless calculate an expected value of $\bar{r}$ for the gamete. The parental origin of segments will alternate across COs on each chromosome in the gamete, and therefore, we can still define, for any given chromosome $k$, the proportions that originate from the two different parents $\left(p_{k}\right.$ and $\left.1-p_{k}\right)$. However, it is not 
possible to know from one chromosome to the next which sets of segments are from the same or different parents. We address this ambiguity by assuming that the probability that the alleles at two loci on separate chromosomes have been shuffled is one-half, consistent with Mendel's Second Law. If there are $n$ chromosomes in the haploid set and chromosome $k$ accounts for a fraction $L_{k}$ of the genome's length and if we determine that a proportion $p_{k}$ of chromosome $k$ is of one parental origin and $1-p_{k}$ is of the other, then

$$
\mathbb{E}[\bar{r}]=\sum_{k=1}^{n} 2 p_{k}\left(1-p_{k}\right) L_{k}^{2}+\frac{1}{2}\left(1-\sum_{k=1}^{n} L_{k}^{2}\right) .
$$

The first term in Eq. 2 is the probability summed over all chromosomes $k$ that two randomly chosen loci are on chromosome $k$ multiplied by the probability that, if so, they are of different parental origin. The second term is the probability that the two randomly chosen loci are on different chromosomes, $1-\sum_{k=1}^{n} L_{k}^{2}$ (i.e., 1 - the probability that a random pair of loci are on the same chromosome), multiplied by $1 / 2$. This second term is probabilistic, so Eq. 2 is an expectation of $\bar{r}$ rather than the value actually realized in the gamete. ${ }^{\ddagger}$

Finally, cytological analysis of meiotic pachytene chromosomes allows to define $\mathrm{CO}$ positions along bivalent chromosomes at that stage of meiotic prophase I. An expected value for $\bar{r}$ can be calculated in this case following the formulas given by Burt et al. (27) and Colombo (81) (Fig. 2A). If the haploid number of chromosomes is $n$ and there are a total of $I$ COs, then these COs divide the bivalents into $n+I$ segments. Label the segments in some (arbitrary) order, and suppose that segment $i$ 's fraction of total genome length is $l_{i}$, with $l_{1}+\cdots+l_{n+I}=1$. For any randomly selected locus pair to shuffle its alleles in the production of a gamete, the two loci need to be situated on different segments, the probability of which is $1-\sum_{i-1}^{n+I} l_{i}^{2}$. If the two loci are indeed situated on different segments, they shuffle their alleles with probability $1 / 2$ [this assumes no chromatid interference $(54,57)]$. Therefore, given the configuration of COs at meiosis I, the probability that the alleles at a randomly chosen pair of loci are shuffled in a resulting gamete is $1 / 2$ multiplied by the probability that the two loci are on separate segments:

$$
\mathbb{E}[\bar{r}]=\frac{1}{2}\left(1-\sum_{i=1}^{n+I} l_{i}^{2}\right) .
$$

$\mathbb{E}[\bar{r}]$, as defined by Eq. 3, is proportional to the Gini-Simpson index (83), a commonly used measure of diversity, especially in ecology (84).

Inter- and Intrachromosomal Components of $\overline{\boldsymbol{r}}$. It is often argued that the predominant source of shuffling in sexual species is IA, or, correspondingly, that the most effective way for a species to increase genome-wide shuffling is to increase the number of chromosomes rather than CO frequency $(1,85,86)$ (Discussion). Our formulation of $\bar{r}$ allows us to partition total shuffling into a component deriving from COs (intrachromosomal shuffling) and a component deriving from IA (interchromosomal shuffling), thereby allowing us to evaluate previous assertions about their relative contributions in a rigorous quantitative way.

\footnotetext{
${ }^{\ddagger}$ Given a gamete sequence and knowledge of the paternal and maternal origins of all chromosome segments, the realized value of $\bar{r}$-being the proportion of locus pairs at which alleles have been shuffled-is precisely known (i.e., here, it is a "parameter"). If the maternal and paternal origins are not known, then $\bar{r}$ becomes a random variable, with its realized value being different for different assortments of the possible parental origins of the various segments. Therefore, we calculate its expectation with respect to the distribution of these possible assortments. Similarly, when we observe COs along bivalent chromosomes at meiosis $\mathrm{I}, \bar{r}$ in a resulting gamete is again a random variable; therefore, we calculate its expected value but this time with respect to the distribution of possible patterns of chromatid involvement in the resolution of COs at meiosis I and segregation patterns of chromatids thereafter.
}

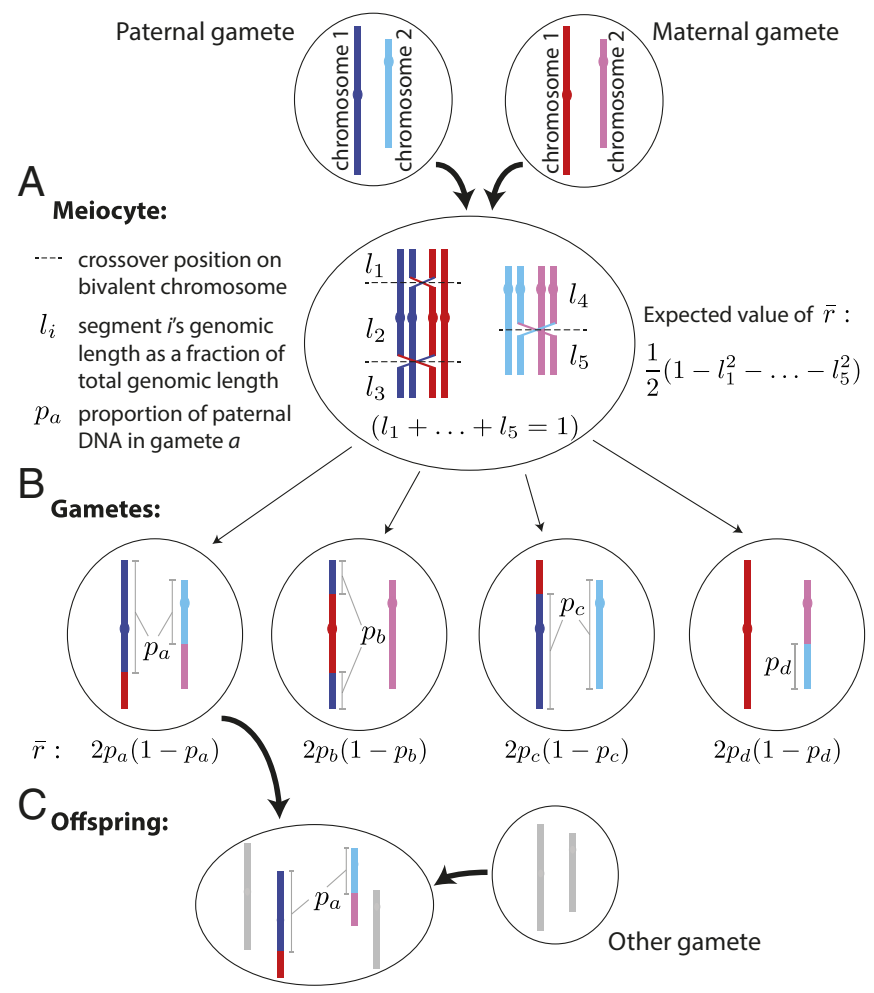

Fig. 2. (A) Calculating the expected value of $\bar{r}, \mathbb{E}[\bar{r}]$, at meiosis I using Eq. 3 . ( $B$ and $C$ ) Calculating $\bar{r}$ in gametes or offspring using Eq. 1.

The interchromosomal component of $\bar{r}$ is the probability that two randomly chosen loci are on separate chromosomes and shuffle their alleles, while the intrachromosomal component is the probability that two loci are on the same chromosome and shuffle their alleles. We first present this decomposition for $\bar{r}$ in a gamete or haploid complement of an offspring. If chromosome $k$ contains a proportion $p_{k}$ of paternal content, then the appropriate decomposition is

$$
\bar{r}=\underbrace{\sum_{k=1}^{n} 2 p_{k}\left(1-p_{k}\right) L_{k}^{2}}_{\text {Intrachrom. component }}+\underbrace{\sum_{i \neq j} 2 p_{i}\left(1-p_{j}\right) L_{i} L_{j}}_{\text {Interchrom. component }} .
$$

If we know $\mathrm{CO}$ realizations in a haploid complement but not the specific parental origin of the segments that they delimit, then we can still calculate a proportion $p_{k}$ of chromosome $k$ to be of one parental origin and $1-p_{k}$ of the other (although not knowing which is which), and therefore, we retain the first term in Eq. 4 but lose information about the second term. The appropriate partition is then

$$
\mathbb{E}[\bar{r}]=\underbrace{\sum_{k=1}^{n} 2 p_{k}\left(1-p_{k}\right) L_{k}^{2}}_{\text {Intrachrom. component }}+\underbrace{\frac{1}{2}\left(1-\sum_{k=1}^{n} L_{k}^{2}\right)}_{\text {Interchrom. component }},
$$

which is the same as Eq. 2.

We now present the decomposition for $\mathbb{E}[\bar{r}]$ at meiosis I. Suppose that the haploid number of chromosomes is $n$ and that bivalent $k$ exhibits $I_{k}$ COs, dividing it into segments $i=1, \ldots, I_{k}+1$ with fractions of total genomic length $l_{k, i}$. Chromosome $k$ 's fraction of total genomic length is $L_{k}=l_{k, 1}+l_{k, 2}+\cdots+l_{k, I_{k}+1}$, with $L_{1}+L_{2}+\cdots+L_{n}=1$. Then, Eq. 3 is partitioned as follows: 


$$
\mathbb{E}[\bar{r}]=\underbrace{\frac{1}{2} \sum_{k=1}^{n}\left(L_{k}^{2}-\sum_{i=1}^{I_{k}+1} l_{k, i}^{2}\right)}_{\text {Intrachrom. component }}+\underbrace{\frac{1}{2}\left(1-\sum_{k=1}^{n} L_{k}^{2}\right)}_{\text {Interchrom. component }} .
$$

Averaging $\bar{r}$. The above measures can be aggregated to obtain the average value of $\bar{r}$ across many gametes (or meiocytes). We denote the average value of a variable $x$ by $\langle x\rangle$. To average $\bar{r}$ given sequence data from many gametes and supposing that, for each gamete, we can distinguish which sequences are paternal and which are maternal, we can take the average value of Eq. 4, noting that the $L_{k}$ are constants:

$$
\langle\bar{r}\rangle=\underbrace{\sum_{k=1}^{n}\left\langle 2 p_{k}\left(1-p_{k}\right)\right\rangle L_{k}^{2}}_{\text {Intrachrom. component }}+\underbrace{\sum_{i \neq j}\left\langle 2 p_{i}\left(1-p_{j}\right)\right\rangle L_{i} L_{j}}_{\text {Interchrom. component }} .
$$

If segregation is Mendelian, then in a large sample of gametes, $\left\langle 2 p_{i}\left(1-p_{j}\right)\right\rangle \approx 1 / 2$, and therefore, the interchromosomal component in Eq. 7 will be close to $\frac{1}{2}\left(1-\sum_{k=1}^{n} L_{k}^{2}\right)$.

If we have sequence data from many gametes and can determine $\mathrm{CO}$ positions but not the parental origin of the sequences that these COs delimit, then we take the average of Eq. 5, noting again that the $L_{k}$ are constants (which here means that the interchromosomal component is constant):

$$
\langle\mathbb{E}[\bar{r}]\rangle=\underbrace{\sum_{k=1}^{n}\left\langle 2 p_{k}\left(1-p_{k}\right)\right\rangle L_{k}^{2}}_{\text {Intrachrom. component }}+\underbrace{\frac{1}{2}\left(1-\sum_{k=1}^{n} L_{k}^{2}\right)}_{\text {Interchrom. component }} .
$$

Given data of $\mathrm{CO}$ positions along bivalent chromosomes in many meiocytes, we take the average of Eq. 6:

$$
\langle\mathbb{E}[\bar{r}]\rangle=\underbrace{\frac{1}{2} \sum_{k=1}^{n}\left(L_{k}^{2}-\left\langle\sum_{i=1}^{I_{k}+1} l_{k, i}^{2}\right\rangle\right)}_{\text {Intrachrom. component }}+\underbrace{\frac{1}{2}\left(1-\sum_{k=1}^{n} L_{k}^{2}\right)}_{\text {Interchrom. component }} .
$$

Eqs. 7-9 mean that we can estimate the average intrachromosomal contribution to $\bar{r}$ separately for each chromosome, possibly from different sets of data, and then combine these averages into a final measure of $\bar{r}$. This is useful, because often in sequencing or cytological studies of large numbers of gametes or meiosis I nuclei, it is possible (or desired) to obtain accurate measurements for only a subset of the chromosomes in each cell so that the sets of cells from which measurements are taken for two chromosomes will not overlap. This is the case, for example, in the rich cytological data from human pachytene nuclei in ref. 48 .

Unrelated to averaging $\bar{r}$ across multiple measurements of individual gametes or meiocytes, an average value for $\bar{r}$ can also be calculated directly given pairwise average rates of shuffling for all loci. These can be estimated from linkage maps generated from pooled sequence data (see below). Suppose that we have measured, for each locus pair $(i, j)$, their rate of shuffling $r_{i j} .\langle\bar{r}\rangle$ is then simply the average value of $r_{i j}$ across all locus pairs $(i, j)$. If $\Lambda$ is the total number of loci, then

$$
\langle\bar{r}\rangle=\sum_{i<j} r_{i j} /\left(\begin{array}{l}
\Lambda \\
2
\end{array}\right)=\underbrace{\sum_{i<j \text { linked }} r_{i j} /\left(\begin{array}{l}
\Lambda \\
2
\end{array}\right)}_{\text {Intrachrom. }}+\underbrace{\sum_{i<j \text { unlinked }} r_{i j} /\left(\begin{array}{l}
\Lambda \\
2
\end{array}\right)}_{\text {Interchrom. }},
$$

where $\left(\begin{array}{c}\Lambda \\ 2\end{array}\right)=\Lambda(\Lambda-1) / 2$. When $r_{i j}=1 / 2$ for all unlinked locus pairs $(i, j)$, the interchromosomal component simplifies to $\left(1-\sum_{k=1}^{n} L_{k}^{2}\right) / 2$ as before.

\section{Properties and Intrinsic Implications of $\overline{\boldsymbol{r}}$}

Properties. We note three properties of $\bar{r}$. First, its minimum value is zero, and its maximum value is one-half, the latter relying on our assumption of many loci. The maximum value of $\bar{r}$ in a gamete can occur by chance equal segregation of maternal and paternal DNA to the gamete. The maximum value of $\mathbb{E}[\bar{r}]$ at meiosis I requires, unrealistically, that every pair of loci either is on separate chromosomes or experiences at least one $\mathrm{CO}$ between them in every meiosis. The minimum value of $\bar{r}$ in a gamete could result from chance segregation of only CO-less chromatids of one parental origin to the gamete. The minimum value of $\mathbb{E}[\bar{r}]$ at meiosis I requires crossing-over to be absent [as in Drosophila males and Lepidoptera females (76)] and either a karyotype of one chromosome or a meiotic process that causes multiple chromosomes to segregate as a single linkage group [as in some species in the evening primrose genus, Oenothera (87)].

Second, $\bar{r}$ satisfies the intuitive property that a $\mathrm{CO}$ at the tip of a chromosome causes less shuffling than a $\mathrm{CO}$ in the middle (some example calculations of $\bar{r}$ are given in Fig. 3). This is easily seen in Eq. 3. Consider a bivalent on which a single $\mathrm{CO}$ will be placed. This will divide the chromosome into two segments of length $l_{1}$ and $l_{2}$, where $l_{1}+l_{2}=L$ is the chromosome's fraction of total genomic length. The contribution of this $\mathrm{CO}$ to $\mathbb{E}[\bar{r}]$ is seen from Eq. 3 to be $1 / 2-$ $\left(l_{1}^{2}+l_{2}^{2}\right) / 2$, which simplifies to $l_{1} l_{2}$ under the constraint $l_{1}+l_{2}=$ L. $l_{1} l_{2}$ is maximized when $l_{1}=l_{2}=L / 2$ (i.e., when the $\mathrm{CO}$ is placed in the middle of the bivalent). It is minimized when $l_{1}=0$ or $l_{2}=0$ (i.e., when the $\mathrm{CO}$ is placed at one of the far ends of the bivalent). In general, as the $\mathrm{CO}$ is moved farther from the middle of the bivalent, $\mathbb{E}[\bar{r}]$ decreases. This is true regardless of the positions of the COs on other chromosomes. Importantly, this relationship also holds if, instead of considering where to place a single $\mathrm{CO}$ on a whole bivalent, we consider where to place a new $\mathrm{CO}$ on a segment already delimited by two COs (or by a CO and a chromosome end). In general, if we place a fixed number of COs along a single chromosome, $\mathbb{E}[\bar{r}]$ is increased if they are evenly spaced (SI Appendix).

Third, $\bar{r}$ will tend to increase sublinearly with both the number of COs and the number of chromosomes (Figs. $3 D-F$ ). For example, increasing the number of COs by some factor will increase the intrachromosomal component of $\bar{r}$ by a lesser factor (and $\bar{r}$ as a whole by a yet lesser factor, because its interchromosomal component is unaffected). Similarly, doubling the number of chromosomes in the haploid set will cause a less than twofold increase in the interchromosomal component of $\bar{r}$. That is, there are "diminishing returns" to genome-wide shuffling from having increasingly more COs and more chromosomes. These effects are described in mathematical detail in SI Appendix.

Intrinsic Implications. We have noted above that, given some number of COs along a chromosome at meiosis $\mathrm{I}, \mathbb{E}[\bar{r}]$ is maximized if they are evenly spaced. This observation carries the interesting implication that positive $\mathrm{CO}$ interference-a classical phenomenon $(88,89)$ where the presence of a $\mathrm{CO}$ at some point along a bivalent chromosome inhibits the formation of nearby COs-will tend to increase $\bar{r}$. It thus suggests a possible selective advantage for this phenomenon (Discussion).

Also, the general diminishing returns to $\bar{r}$ of having more COs (above) carry particular implications for differences in total autosomal shuffling in the two sexes of a species. When the number of COs and/or their localization do not differ grossly between the sexes, then the amount of shuffling will be similar in both sexes (e.g., for male and female humans as discussed below).

\section{Experimental Determination of CO Positions}

Measurement of $\bar{r}$ requires that CO positions along chromosomes can be accurately determined. This was previously not possible, but cytological advances have made it possible to efficiently and accurately visualize the positions of COs on pachytene bivalent chromosomes $(37,74,75,90,91)$, while rapid technological advances in DNA sequencing have allowed CO positions to be 
A

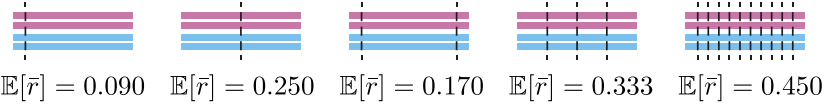

B

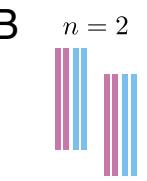

$\mathbb{E}[\bar{r}]=0.250$

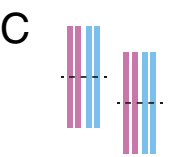

$\mathbb{E}[\bar{r}]=0.375$

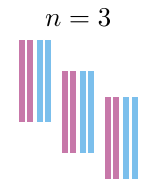

$\mathbb{E}[\bar{r}]=0.333$

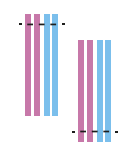

$\mathbb{E}[\bar{r}]=0.295$ $n=10$

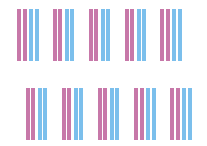

$\mathbb{E}[\bar{r}]=0.450$

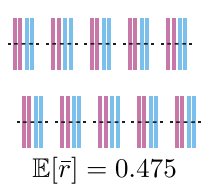

$n=23$

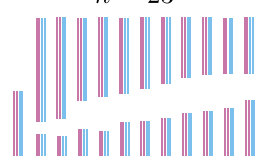

$\mathbb{E}[\bar{r}]=0.474$

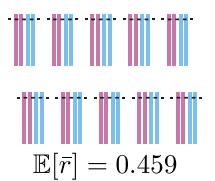

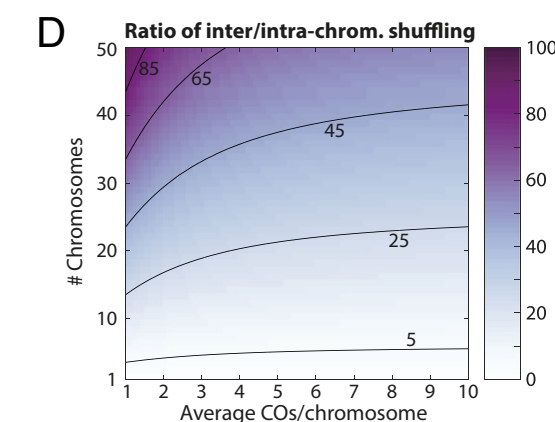
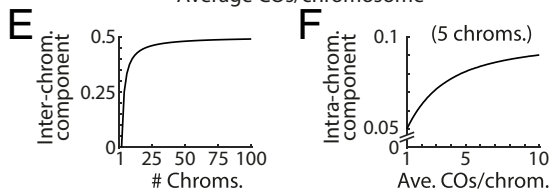

Fig. 3. (A-C) Example values of the expected value of $\bar{r}, \mathbb{E}[\bar{r}]$, at meiosis I. In each case, $\mathbb{E}[\bar{r}]$ is calculated according to Eq. 3. (A) Various $C O$ numbers and positions in a diploid organism with only one chromosome. Notice that $\mathbb{E}[\bar{r}]$ increases sublinearly with $\mathrm{CO}$ number, even when $\mathrm{COs}$ are evenly spaced so as to maximize $\mathbb{E}[\bar{r}]$. (B) Various chromosome numbers for a diploid organism without crossing-over. Notice that $\mathbb{E}[\bar{r}]$ increases sublinearly with chromosome number, even when chromosomes are evenly sized so as to maximize $\mathbb{E}[\bar{r}]$. Notice too that, when the number of chromosomes is not small, $\mathbb{E}[\bar{r}]$ is close to its maximum value of one-half. Column 4 represents a CO-less meiosis in a human female, in which case $\mathbb{E}[\bar{r}]$ is 0.474 . (C) Various configurations of COs in a diploid organism with multiple chromosomes. $(D)$ The ratio of contributions to $\mathbb{E}[\bar{r}]$ from IA (interchromosomal shuffling) and COs (intrachromosomal shuffling). Isoclines indicate $\mathrm{CO}$ and chromosome number combinations across which this ratio is constant. For example, the " 45 " isocline indicates where IA contributes 45 times more shuffling than COs do. Chromosomes are assumed to be of equal size, and COs are assumed always to be evenly spaced along chromosomes. For each combination of $n \geq 2$ chromosomes in the haploid set and $l \geq 1$ COs per chromosome on average, we simulate $10^{5}$ nuclei. For each of the $n$ bivalent chromosomes in a nucleus, we draw a value from a Poisson distribution with parameter $I-1$ and add it to 1 mandatory CO to get the total number of COs for that chromosome. We then calculate the average total intrachromosomal contribution to $\mathbb{E}[\bar{r}]$ across the nuclei and compare it with the interchromosomal contribution (which is constant across nuclei). When the number of chromosomes is not small, the interchromosomal component dominates. (E) The interchromosomal component increases at a decreasing rate as chromosome number increases. $(F)$ Similarly, holding constant the number of chromosomes (here, five), the intrachromosomal component increases at a decreasing rate as average CO number increases.

determined at a fine genomic scale using sequence/marker analysis of pedigrees $(40,41,92,93)$, individual gametes $(94,95)$, and meiotic triads/tetrads (42, 96-98). These technological advances allow simple estimation of $\bar{r}$.

Cytological Analysis. The physical positions of COs along the axes of pachytene chromosomes can be determined reliably in spread pachytene nuclei (or in 3D reconstructions from serial sectioning) either with electron microscopy by direct visualization of "late recombination nodules" [which mark all COs (99-102)] or with light microscopy using immunofluorescent staining techniques to detect "type I" (interfering) COs (reviewed in ref. 103) (Fig. 4), which are the vast majority in most organisms (104). The latter method is now most commonly used.

Visualization of the chromosome axes can be achieved by staining either the axes or the synaptonemal complex central region (e.g., SYCP1-3). CO positions along the axes can be visualized by staining molecules specific to $\mathrm{CO}$ recombination complexes (e.g., MLH1). If desired, the positions of centromeres along the axes can be visualized by staining for centromeric proteins, such as CENP-A, and individual chromosomes can be identified by FISH, locus-specific fluorescent tags (e.g., fluorescent repressor-operator system arrays), or in favorable cases [e.g., Arabidopsis (105)], relative chromosome length. Spread pachytene nuclei immunostained for $\mathrm{CO}$ positions are now common in the literature and have been generated for many species (e.g., 28 bovid species in ref. 106).

Only physical distances (in micrometers) along chromosomes can be directly inferred from cytological data, so the measurement of $\bar{r}$ from such data requires a way to convert physical distances into genomic distances (in base pairs). The use of pachytene-stage chromosomes (Fig. 4) is particularly advantageous in this regard, because at this stage of meiosis, genomic distance is proportional to physical distance along the chromosome axes (below and SI Appendix).

The physical structure of these chromosomes is highly regular: the chromatin of each chromatid is arranged in a linear array of loops, the bases of which are at regular intervals along a common axis (reviewed in refs. 101, 107, and 108). Almost all of the DNA is accommodated in the loops, with very little DNA in the axis between loop bases (101, 107-109). Therefore, the genomic lengths of the loops directly define the genomic distance per unit physical distance along a pachytene bivalent axis (the local "packing ratio") $(101,106,108,110,111)$. Moreover, the local packing ratio along and across different chromosomes is approximately constant within each nucleus, at least at the relatively crude scale of inter-CO distances required for analysis of $\bar{r}$, as indicated by two criteria. First, physical loop lengths can be measured directly and, in a variety of species, appear to vary minimally along and across chromosomes within nuclei (refs. 107 and 111-114; reviewed in ref. 101). Second, the relative genomic lengths of chromosomes closely match their relative physical axis lengths at pachytene (SI Appendix, Fig. S2 shows examples from diverse species), from which we infer that the average packing ratio of different chromosomes within a nucleus is approximately constant. Given these considerations, the chromosome segments' fractions of total genomic length (base pairs), as required for calculation of $\mathbb{E}[\bar{r}]$, correspond to, and thus can be defined by their fractions of total physical axis length (micrometers). Therefore, their fractions of total axis length (easily measured from cytological spreads) can be substituted into the formulas derived above for $\mathbb{E}[\bar{r}] .^{\S}$

Sequence Analysis. The genomic positions of COs in an individual's meioses can be inferred by sequencing the individual products of those meioses-either directly by sequencing individual gametes or polar bodies or by inferring gametic genomes by sequencing individual diploid offspring-provided that the

\footnotetext{
$\S$ In some species [e.g., tomato (115)], it has been reported that loops are longer in heterochromatin than in euchromatin. While the data in SI Appendix, Fig. S2 suggest that this is not general, in such species appropriate adjustments would be needed to convert physical lengths to genomic lengths.
} 


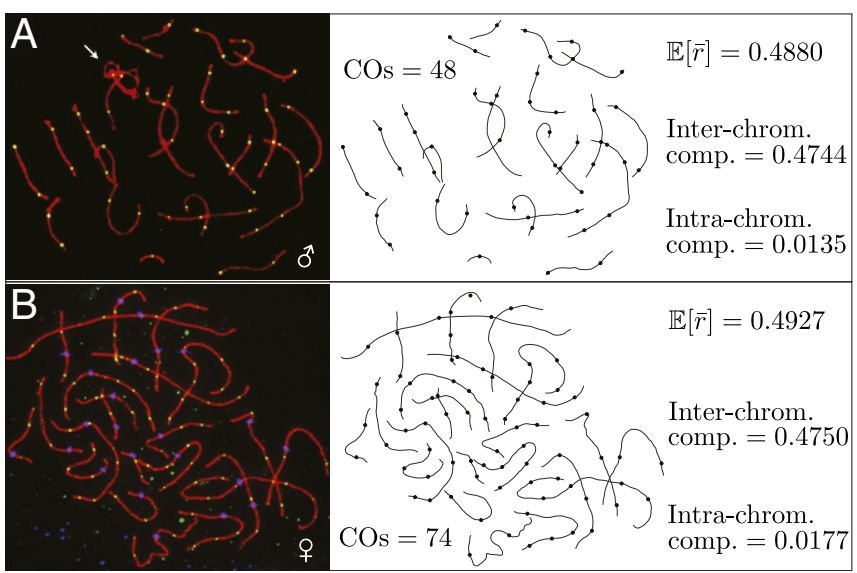

Fig. 4. Calculation of $\mathbb{E}[\bar{r}]$ from $\mathrm{CO}$ patterns at meiosis I in humans. Left shows micrographs of immunostained spreads of a pachytene spermatocyte $(A)$ and a pachytene oocyte $(B)$. The red lines show localization of the synaptonemal complex protein SYCP3 and demarcate the structural axes of the chromosomes. The green dots along the axes show foci of the protein MLH1 and demarcate the positions of $\mathrm{CO}$ associations along the axes. The white arrow in $A$ points to the paired $\mathrm{X}$ and $\mathrm{Y}$ chromosomes. In $B$, the blue dots along the chromosome axes mark the centromeres. The paired $\mathrm{X}$ chromosomes in $B$ have not been identified and are included in the calculation of $\mathbb{E}[\bar{r}]$. A, Center and $B$, Center show the traced axes and $C O$ positions from the spreads. From these, the lengths of the segments separated by the COs can be measured and converted to fractions of the total physical axis length (these measurements can be found in Dataset S1). The segments' fractions of total physical axis length are approximately equal to their fractions of total genomic length for reasons described in the text. With these fractions of genomic length, $\mathbb{E}[\bar{r}]$ may be calculated for each spread according to Eq. 3, and its inter- and intrachromosomal components can be determined according to Eq. 6 ( $A$, Right and $B$, Right). Notice that we do not require individual chromosomes to be identified to calculate $\mathbb{E}[\bar{r}]$ or its intra- and interchromosomal components. $(A)$ Modified from ref. 103 by permission of Oxford University Press. (B) Modified from ref. 39.

individual's diploid genome has been haplophased. Haplophasing can be achieved by sequencing an extended pedigree involving the individual $(40,73,116)$; by sequencing multiple offspring, gametes, and/or polar bodies of the individual $(42,52,95,97,117$, 118 ); or by isolating individual chromosomes from a somatic cell and sequencing them separately $(94,119)$.

$\bar{r}$ can also be calculated from linkage maps derived from pooled sequencing data [as obtained, for example, from population pedigrees (40) or pooled sperm (120)]. A linkage map gives the map distance (in centimorgans) between pairs of linked markers. Using this, we can generate a grid of evenly spaced loci for each chromosome (to ensure even sampling of loci in the calculation of $\bar{r}$ ) and impute map distances between these by linear interpolation from distances between true markers in the linkage map (details are in SI Appendix). Thus, we generate a map distance $d_{i j}$ between every pair of linked loci $(i, j)$, which we can convert to a rate of shuffling $r_{i j}$ using a map function (54) [e.g., Kosambi's (121): $\left.r_{i j}=r\left(d_{i j}\right)=\frac{1}{2} \tanh \left(2 d_{i j}\right)\right]$. This gives a rate of shuffling for every linked locus pair, from which we calculate the average intrachromosomal component of $\langle\bar{r}\rangle$ using Eq. 10.

\section{Measuring $\bar{r}$ in Humans}

Analysis of Cytological Data. Fig. 4 shows the calculation of $\mathbb{E}[\bar{r}]$ from the immunostained pachytene chromosomes of a single human spermatocyte (103) and oocyte (39). For both spermatocyte and oocyte, $\mathbb{E}[\bar{r}]$ is close to its maximum value of $1 / 2$. This high level of shuffling is due almost entirely to IA: in both cases, the interchromosomal component of $\mathbb{E}[\bar{r}]$ is much larger than the intrachromosomal component (from COs) - by a factor of about 35 in the male and 27 in the female.

The spermatocyte in Fig. $4 A$ contains easily identified, partially paired $\mathrm{X}$ and $\mathrm{Y}$ chromosomes, while the oocyte in Fig. $4 B$ contains a bivalent of paired $\mathrm{X}$ chromosomes, which, in this case, has not been distinguished from the other bivalents (e.g., by FISH). Therefore, the $\mathrm{X}$ is included in the calculation for the oocyte, so the interchromosomal components of $\mathbb{E}[\bar{r}]$ for the spermatocyte and oocyte are not expected to be equal. Although we have estimated them directly from the single cytological spreads in Fig. 4, they can be calculated exactly from known genomic lengths of the chromosomes. Substituting the chromosome lengths reported in assembly GRCh38.p11 of the human reference genome (available online at https://www.ncbi. nlm.nih.gov/grc/human/data?asm=GRCh38.p11) (lengths are listed in Dataset S1) into the first term in Eq. 6, we find that the value for the spermatocyte should be 0.4730 , close to the value of 0.4744 calculated in Fig. $4 A$, while the value for the oocyte should be 0.4744 , close to the value of 0.4750 calculated in Fig. $4 B$. These close matches illustrate the reliability of physical length as a proxy for genomic length in the calculation of $\mathbb{E}[\bar{r}]$.

Interestingly, although the female spread exhibits about $50 \%$ more COs than the male spread (74 vs. 48 ), the intrachromosomal component of $\mathbb{E}[\bar{r}]$ for the female spread is only about $30 \%$ larger than that of the male spread. This is expected from the general arguments given earlier concerning the diminishing returns of more COs (Fig. $3 D$ and $F$ ).

We have carried out measurement of $\mathbb{E}[\bar{r}]$ using data from 824 spermatocytes from 10 male humans (data from ref. 122) (provided by Fei Sun, Nantong University, Nantong, China). Using Eq. 9, we calculate each chromosome's average contribution to the intrachromosomal component of $\mathbb{E}[\bar{r}]$ (SI Appendix, Table S1). Summing these values, we find that the average intrachromosomal component of $\mathbb{E}[\bar{r}]$ is 0.0143 , similar to the value calculated for the single spermatocyte in Fig. $4 A$. Restricting attention to the 715 cells with data for every chromosome allows us to calculate the average and SD of the intrachromosomal component of $\mathbb{E}[\bar{r}]$ for each of the 10 individuals. The SD for each individual is of order 0.0015 (SI Appendix, Table S2) or about $10 \%$ of the average value. There is some variation among the individuals for the average value of the intrachromosomal component of $\mathbb{E}[\bar{r}]$ : the minimum is 0.0130 , while the maximum is 0.0153. Interestingly, the minimum and maximum do not come from the individuals with the smallest and largest average $\mathrm{CO}$ frequencies, respectively (SI Appendix, Table S2). In fact, the individual with the largest average value of the intrachromosomal component of $\mathbb{E}[\bar{r}]$ has only the 4th largest average number of COs per spermatocyte. This further highlights the importance of $\mathrm{CO}$ position for the amount of genetic shuffling and hints that individuals might differ systematically in their CO positioning in ways that affect the amount of shuffling in their gametes.

Analysis of Gamete Sequence Data. Hou et al. (97) sequenced the products of multiple meioses (first polar bodies, second polar bodies, and ova) of several females and haplophased these females directly from the sequences, allowing the $\mathrm{CO}$ points in those products to be determined. Fig. 5 is a modified version of figure S2 in ref. 97, showing the CO points along the chromosomes of an egg cell from one of the individuals. Because relatives of the individual were not sequenced, the paternal and maternal sequences cannot be discerned. Therefore, calculation of $\mathbb{E}[\bar{r}]$ proceeds according to Eq. 2, with its inter- and intrachromosomal components determined according to Eq. 5. Carrying out this calculation for all chromosomes, including the $\mathrm{X}$, yields $\mathbb{E}[\bar{r}]=0.4971$, with an interchromosomal component of 0.4756 (calculated using chromosome lengths as they appear in Fig. 5) and an intrachromosomal component of 0.0215 (all details are in Dataset S2). The interchromosomal contribution is about 22 times larger than the intrachromosomal contribution. Excluding the $\mathrm{X}, \mathbb{E}[\bar{r}]=0.4965$, with an interchromosomal component of 0.4735 and an intrachromosomal component of 0.0230 .

Fan et al. (119) haplophased a male using microfluidic techniques, and Wang et al. (94) sequenced 91 individual sperm cells from this male using the haplophasing from ref. 119 to determine the $\mathrm{CO}$ points in each sperm's sequence (given in table S2 in 
ref. 94). Because maternal and paternal sequences are not known, we use Eq. 8 to calculate the average value across all 91 gametes of $\mathbb{E}[\bar{r}]$ and its components. This yields an average value of $\mathbb{E}[\bar{r}]$ of 0.4856 , with average inter- and intrachromosomal components of 0.4729 and 0.0127 , respectively (MATLAB code for all calculations is available from https://github.com/cveller/rbar). The average interchromosomal contribution is about 37 times larger than the average intrachromosomal contribution. In calculating the interchromosomal component, because some shuffled SNP positions for this individual exceeded the chromosome lengths of the human reference genome, we used the position of the last SNP on each chromosome (as reported in ref. 119, dataset S2) as our proxy of that chromosome's genomic length. Because there is no information about the parental origin of the sequences in each sperm, the interchromosomal component for each sperm is an average, deduced from the fixed genomic lengths of the individual's chromosomes (using Eq. 5). There is, therefore, no variance across gametes in this component. The $\mathrm{SD}$ of the intrachromosomal component of $\bar{r}$ across gametes is 0.0026 (coefficient of variation $\approx 0.2$ ). The minimum and maximum values of the intrachromosomal component across all 91 sperm are 0.0058 and 0.0178 , respectively. The average intrachromosomal component for this individual is lower than that calculated from spermatocytes in the previous subsection (0.0143). This is expected, as he is homozygous for a variant of the RNF212 gene that, in homozygous form, is associated with a reduction of $\sim 5-7.5 \%$ in $\mathrm{CO}$ numbers in males $(118,123)$. Indeed, the 91 sperm show, on average, $22.8 \mathrm{COs}$ each (minimum, 12; maximum, 32) compared with the previously cited averages of 25.9 for Icelandic males (52) and 26.2 for Hutterite males (117).

Analysis of Linkage Map Data. Kong et al. (41) sequenced 8,850 mother-offspring and 6,407 father-offspring pairs of Icelandic individuals to generate high-density linkage maps for both sexes (data are available at https://www.decode.com/addendum). For each sex, we apply the method described above for generating a linkage map of evenly spaced "pseudomarker" loci and estimate rates of shuffling for all linked locus pairs from the map distances between them using the Kosambi map function (MATLAB code is available from https://github.com/cveller/ rbar). We then estimate the intrachromosomal component of $\langle\bar{r}\rangle$ using Eq. 10 (the interchromosomal component is calculated from known chromosome lengths). This yields a male value of 0.0138 , similar to the value of 0.0143 calculated above from 824 spermatocytes (the chromosome-specific contributions are similar as well) (SI Appendix, Table S4). This agreement validates the linkage map method of estimating $\langle\bar{r}\rangle$. The female value, including the $\mathrm{X}$ chromosome, is 0.0183 , similar to the value of 0.0177 calculated from the single-oocyte spread in Fig. 4 but smaller than the value of 0.0215 calculated from the single egg in Fig. 5. Excluding the $\mathrm{X}$, the female value is 0.0193 .

\section{Discussion}

The most common current measures of genome-wide genetic shuffling-CO frequency and map length-do not take into account the positions of COs. This is a significant limitation, because terminal COs cause less shuffling than central COs. Traditional measures of aggregate shuffling also do not take into account IA. Here, we have proposed a measure of genome-wide shuffling that naturally takes both of these features into account. This measure, $\bar{r}$, is the probability that a randomly chosen pair of loci shuffles their alleles in meiosis.

$\bar{r}$ Should Be the Measure of Choice for Evaluation and Comparison of Genetic Shuffling. $\bar{r}$ is the most direct measure of the aggregate amount of shuffling that occurs in the production of gametes. Application of this measure should, therefore, significantly improve the possibility of understanding the nature, basis, and significance of genetic shuffling and its variation across different situations (e.g., across species). We hope that this will become common practice where possible. In support of this possibility, we have demonstrated that $\bar{r}$ can readily be measured from standard sequence and cytological data.

Distinguishing the Contributions of Crossing-Over and Mendel's Second Law. We have shown that $\bar{r}$ can be decomposed into a component from COs and a component from IA. The relative importance of COs and IA as sources of shuffling has long been discussed $(1,15,85,86)$. Theories of the adaptive value of shuffling fall roughly into two categories: those positing a short-term advantage [offspring diversification; e.g., the Tangled Bank (1, $124)$ and sib-competition $(2,125)$ theories] and those positing a long-term advantage [e.g., the "Fisher-Muller" theory that gradually reducing allelic correlations over time allows natural selection to operate more efficiently on genes at different loci (3-7)]. Burt (86) has argued that COs are more effective than IA in gradually reducing allelic associations (because IA shuffles genomes at fixed points, whereas COs can occur at many points along a chromosome). Under this view, the CO component of $\bar{r}$ would be more important than the IA component for long-term theories of the adaptive value of shuffling. However, the short-term theories rely on aggregate shuffling per se. We have shown, using humans as an example, that IA will typically be the greatest contributor to aggregate shuffling. Therefore, the IA component of $\bar{r}$ may be more important than the $\mathrm{CO}$ component for short-term theories of the adaptive purpose of shuffling. Our decomposition of $\bar{r}$ into its separate components allows these distinctions to be tested quantitatively.

Co Interference Increases Genetic Shuffling. A property of the intrachromosomal component of $\bar{r}$ is that it increases as COs become more evenly spread out. Thus, intriguingly, interference among COs will tend to increase the amount of shuffling that they cause [similar points have been made by Goldstein et al. (126), and Gorlov and Gorlova (127)]. In SI Appendix, we quantify this effect for human males using the spermatocyte data from ref. 122 described above. Following the method used in refs. 94 and 96 for sampling COs without interference, for each chromosome we calculate an interferenceless average contribution to intrachromosomal $\mathbb{E}[\bar{r}]$ by resampling, in each spermatocyte, the positions of the COs independently from the empirical distribution pooled across all spermatocytes. The observed average

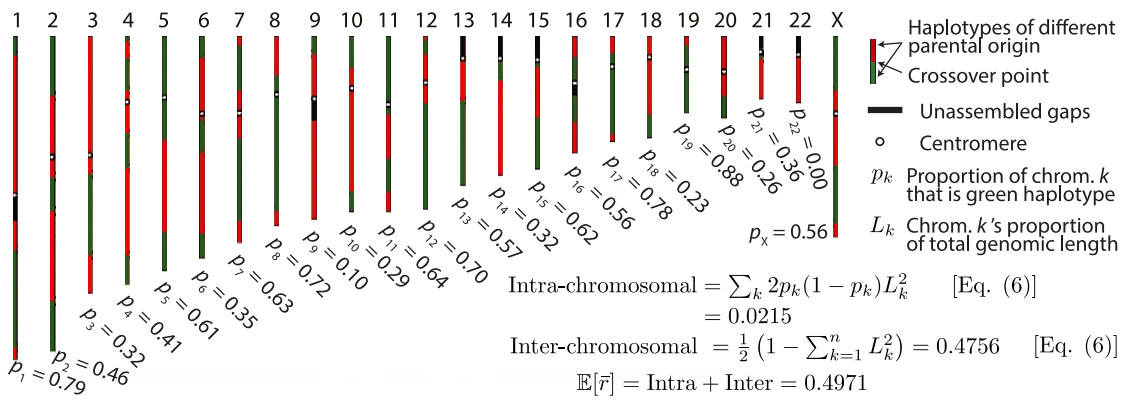

Fig. 5. Calculation of $\bar{r}$ from the sequence of an individual egg obtained from a haplophased human female. With no sequencing of an extended pedigree, we cannot tell which of the haplotypes separated by $\mathrm{CO}$ points are maternal and which are paternal. Therefore, we cannot directly calculate $p$, the proportion of the egg's DNA that is paternal. We can, however, for each chromosome $k$ find the proportion $p_{k}$ that is of one parental origin (without knowing which) and then use Eq. 5 to calculate $\bar{r}$ and its components. Modified from ref. 97. Copyright (2013), with permission from Elsevier. 
value of the intrachromosomal contribution to $\mathbb{E}[\bar{r}]$ is about $15 \%$ higher than this interferenceless value.

$\mathrm{CO}$ interference, first described more than a century ago (88), is a deeply conserved feature of the meiotic program $(128,129)$. Despite this, its adaptive function, if any, remains unclear $(104,130,131)$. One category of hypotheses invokes mechanical advantages of spread-out COs either to bivalent stability in prophase (132) or to homolog segregation at meiosis I (133). However, such ideas are challenged by the fact that meiotic segregation proceeds without trouble in organisms that lack CO interference [e.g., fission yeast and Aspergillus (134)] or that have had interference experimentally reduced or eliminated $(135,136)$. This study raises the possibility of a qualitatively different idea: that $\mathrm{CO}$ interference provides an evolutionary advantage via its effects on genetic transmission. By explicitly taking into account the positions of COs in quantifying how much shuffling they cause, we show that $\mathrm{CO}$ interference serves to increase the shuffling caused by a given number of COs. This finding, therefore, suggests a possible adaptive function of $\mathrm{CO}$ interference.

Additional Measures of Genetic Shuffling. $\bar{r}$ is a suitable measure of the total amount of shuffling, linearly aggregating the rates of shuffling between different locus pairs. In this way, it quantifies the first-order effect of shuffling: the genetic diversification of gametes/offspring (1). However, certain population genetic properties exhibit nonlinear dependence on the rates of shuffling between loci. For example, theoretical studies have determined that certain allelic associations can jointly increase in frequency only if their constituent loci shuffle their alleles at a rate below some critical threshold. This has been shown for coadapted gene complexes exhibiting positive fitness epistasis (137-139), "poison-antidote" gamete-killing haplotypes involved in meiotic drive $(11,140-142)$, and associations between sex-determining genes and genes with sexually antagonistic fitness effects (53, 143-145). Similarly, Hill-Robertson interference is effective only among loci that are tightly linked, shuffling their alleles at a rate below a small threshold (which depends on the effective population size and other parameters) (146). In quantifying these various properties at an aggregate level, a more suitable measure would be the proportion of locus pairs that shuffle their alleles at a rate below the critical threshold. Such measures would be informative, for example, of which chromosomes are most likely to be co-opted as new sex chromosomes, of how susceptible a species is to the invasion of meiotic drive complexes (or within species, which chromosomes are most likely to harbor such complexes), or of the average fitness reduction caused by Hill-Robertson interference. Calculating these threshold-based aggregate measures would require estimates of the average rates of shuffling for specific locus pairs. High-density linkage maps would, therefore, be especially promising for such measurements.

Taking Gene Density into Account. The calculation of $\bar{r}$, as we have defined it, treats all regions of equal genomic length as of the same importance. However, the quantity that we seek to measure is really the amount of shuffling of functional genetic elements - it is largely irrelevant if two functionless pseudogenes shuffle, for example. Correcting the calculation of $\bar{r}$ to take gene density into account is an important extension, a point already noted by Burt et al. (27). This is especially so if the distribution of genes is nonrandom along or across chromosomes. Correcting for gene densities of different genomic regions

1. Bell G (1982) The Masterpiece of Nature: The Evolution and Genetics of Sexuality (University of California Press, Berkeley, CA).

2. Williams GC (1975) Sex and Evolution (Princeton Univ Press, Princeton).

3. Fisher RA (1930) The Genetical Theory of Natural Selection (Clarendon, Oxford).

4. Muller HJ (1932) Some genetic aspects of sex. Am Nat 66:118-138.

5. Muller HJ (1964) The relation of recombination to mutational advance. Mut Res 1: 2-9.

6. Hill WG, Robertson A (1966) The effect of linkage on limits to artificial selection. Genet Res 8:269-294. would be easiest with sequence data, especially in species with well-annotated genomes. For species without well-annotated genomes, proxies of regional gene density, such as euchromatin content or guanine-cytosine richness, could be used. Correcting for gene density with cytological data would be more complicated, since it requires knowledge of the identity and orientation of the various chromosomes. At a crude resolution, this can be achieved with chromosome-specific FISH probes (and if required, centromere localization). Finer-scale identification of different genomic regions in cytological spreads could be achieved using high-resolution FISH (147-149). In any case, the usual problems would arise in deciding which parts of the genome are "functional" $(150,151)$.

Taking Heterozygosity into Account. Genetic shuffling of alleles at two loci in meiosis is practically relevant only if both loci are heterozygous. A simple modification of $\bar{r}$ that measures the extent of such "effective" shuffling in meiosis is the proportion of locus pairs that shuffle their alleles and are both heterozygous. Again, we are mostly interested in the shuffling of functional elements, in which case estimation of this measure requires knowledge of the genomic landscape of functional heterozygosity [e.g., nonsynonymous heterozygosity in protein-coding genes as available for humans (152)] together with knowledge of linkage disequilibrium between nearby loci [e.g., for humans (153)].

Taking Gene Conversion into Account. Another source of shuffling, in addition to COs and IA, is gene conversion (GC) (154, 155): the unidirectional copying of a DNA tract from one chromatid (the donor) to a homologous chromatid (the acceptor) either in mitosis or in meiosis. In SI Appendix, we show how to take both mitotic and meiotic GC into account in measuring $\bar{r}$. In meiosis, chromosome pairing involves many points of interaction between homologous chromatids $[\sim 200$ across all chromosomes in mouse spermatocytes (156)], each induced by a double-strand break on one of the chromatids. In most organisms, only a minority of these interactions result in COs $(104,157,158)$, the majority instead resulting in non-COs. Both outcomes are associated with short tracts of GC [e.g., $\sim 100$ bp for non-CO GCs and $\sim 500$ bp for CO GCs in mice (98, 156)]. These tracts, although numerous, are too short to seriously affect the total amount of shuffling [for example, the contribution from meiotic GCs in mammals will be of the order 10,000 times smaller than the contribution from COs (SI Appendix); in yeast, this figure is smaller-about 200 (SI Appendix)]; however, they may have important local effects $(154,159)$. Mitotic GC tracts result from homologous chromosome repair, are typically much longer than meiotic GC tracts (often of order $10 \mathrm{~kb})(160,161)$, and if they occur in germline cell divisions leading to meiocytes, can lead to shuffling of maternal and paternal DNA in gametes (which should especially affect males, who have more germline cell divisions). Too little is presently known about the global frequency of these events for us to give a reasonable quantitative estimate of how much shuffling they cause.

ACKNOWLEDGMENTS. We thank Anthony Geneva, David Haig, Arbe Harpak, Dan Hartl, Alison Hill, Thomas Lenormand, Pavitra Muralidhar Naomi Pierce, Molly Przeworski, Nadia Singh, and Martin White for helpfu comments and Colin Donihue for help with figure preparation. C.V. thanks Bob Trivers for impressing on him the importance of CO position for the amount of recombination. The research of N.K. was supported by NIH Grant GM-044794.

7. Felsenstein J (1974) The evolutionary advantage of recombination. Genetics 78:737756.

8. McDonald MJ, Rice DP, Desai MM (2016) Sex speeds adaptation by altering the dynamics of molecular evolution. Nature 531:233-236.

9. Hamilton WD (1980) Sex versus non-sex versus parasite. Oikos 35:282-290.

10. Hamilton WD, Axelrod R, Tanese R (1990) Sexual reproduction as an adaptation to resist parasites (a review). Proc Natl Acad Sci USA 87:3566-3573.

11. Haig D, Grafen A (1991) Genetic scrambling as a defence against meiotic drive. $J$ Theor Biol 153:531-558. 
12. Burt A, Trivers RL (2006) Genes in Conflict (Belknap, Cambridge, MA).

13. Haig D (2010) Games in tetrads: Segregation, recombination, and meiotic drive. Am Nat 176:404-413.

14. Brandvain Y, Coop G (2012) Scrambling eggs: Meiotic drive and the evolution of female recombination rates. Genetics 190:709-723.

15. Burt A, Bell G (1987) Mammalian chiasma frequencies as a test of two theories of recombination. Nature 326:803-805.

16. True JR, Mercer JM, Laurie CC (1996) Differences in crossover frequency and distribution among three sibling species of Drosophila. Genetics 142:507523.

17. Ross-lbarra J (2004) The evolution of recombination under domestication: A test of two hypotheses. Am Nat 163:105-112.

18. Dumont BL, Payseur BA (2008) Evolution of the genomic rate of recombination in mammals. Evolution 62:276-294.

19. Dumont BL, Payseur BA (2011) Evolution of the genomic recombination rate in murid rodents. Genetics 187:643-657.

20. Segura J, et al. (2013) Evolution of recombination in eutherian mammals: Insights into mechanisms that affect recombination rates and crossover interference. Proc Roy Soc B Biol Sci 280:20131945.

21. Brion C, et al. (2017) Variation of the meiotic recombination landscape and properties over a broad evolutionary distance in yeasts. PLoS Genet 13:e1006917.

22. Smukowski CS, Noor MAF (2011) Recombination rate variation in closely related species. Heredity 107:496-508.

23. Stapley J, Feulner PGD, Johnston SE, Santure AW, Smadja CM (2017) Variation in recombination frequency and distribution across eukaryotes: Patterns and processes. Philos Trans R Soc B Biol Sci 372:20160455.

24. Dapper AL, Payseur BA (2017) Connecting theory and data to understand recombination rate evolution. Philos Trans $R$ Soc B Biol Sci 372:20160469.

25. Wilfert L, Gadau J, Schmid-Hempel P (2007) Variation in genomic recombination rates among animal taxa and the case of social insects. Heredity 98:189-197.

26. Trivers RL (1988) Sex differences in rates of recombination and sexual selection. The Evolution of Sex: An Examination of Current Ideas, eds Michod RE, Levin BR (Sinauer, Sunderland, MA), pp 270-286.

27. Burt A, Bell G, Harvey PH (1991) Sex differences in recombination. J Evol Biol 4:259 277.

28. Lenormand T (2003) The evolution of sex dimorphism in recombination. Genetics 163:811-822.

29. Lenormand T, Dutheil J (2005) Recombination difference between sexes: A role for haploid selection. PLoS Biol 3:e63.

30. Kidwell MG (1972) Genetic change of recombination value in Drosophila melanogaster. I. Artificial selection for high and low recombination and some properties of recombination-modifying genes. Genetics 70:419-432.

31. Kidwell MG (1972) Genetic change of recombination value in Drosophila melanogaster. II. Simulated natural selection. Genetics 70:433-443.

32. Comeron JM, Ratnappan R, Bailin S (2012) The many landscapes of recombination in Drosophila melanogaster. PLoS Genet 8:e1002905.

33. Hunter C, Singh N (2014) Do males matter? Testing the effects of male genetic background on female meiotic crossover rates in Drosophila melanogaster. Evolution 68:2718-2726.

34. Hunter C, Huang W, Mackay T, Singh N (2016) The genetic architecture of natural variation in recombination rate in Drosophila melanogaster. PLoS Genet 12:e1005951.

35. Koehler KE, Cherry JP, Lynn A, Hunt PA, Hassold TJ (2002) Genetic control of mammalian meiotic recombination. I. Variation in exchange frequencies among males from inbred mouse strains. Genetics 162:297-306.

36. Dumont BL, Broman KW, Payseur BA (2009) Variation in genomic recombination rates among heterogeneous stock mice. Genetics 182:1345-1349.

37. Lynn A, et al. (2002) Covariation of synaptonemal complex length and mammalian meiotic exchange rates. Science 296:2222-2225.

38. Kong A, et al. (2004) Recombination rate and reproductive success in humans. Nat Genet 36:1203-1206.

39. Cheng EY, et al. (2009) Meiotic recombination in human oocytes. PLoS Genet 5:e1000661.

40. Broman KW, Murray JC, Sheffield VC, White RL, Weber JL (1998) Comprehensive human genetic maps: Individual and sex-specific variation in recombination. $A m$ Hum Genet 63:861-869.

41. Kong A, et al. (2010) Fine-scale recombination rate differences between sexes, populations and individuals. Nature 467:1099-1103.

42. Ottolini CS, et al. (2015) Genome-wide maps of recombination and chromosome seg regation in human oocytes and embryos show selection for maternal recombination rates. Nat Genet 47:727-735.

43. Sanchez-Moran E, Armstrong S, Santos J, Franklin F, Jones G (2002) Variation in chiasma frequency among eight accessions of Arabidopsis thaliana. Genetics 162:1415-1422.

44. Ritz KR, Noor MAF, Singh ND (2017) Variation in recombination rate: Adaptive or not? Trends Genet 33:364-374.

45. Maudlin I (1972) Developmental variation of chiasma frequency in Chorthippus brunneus. Heredity 29:259-262.

46. Hassold T, et al. (2004) Cytological studies of meiotic recombination in human males. Cytogen Genome Res 107:249-255.

47. Lenzi ML, et al. (2005) Extreme heterogeneity in the molecular events leading to the establishment of chiasmata during meiosis I in human oocytes. Am J Hum Genet 76:112-127.
48. Gruhn JR, Rubio C, Broman KW, Hunt PA, Hassold T (2013) Cytological studies of human meiosis: Sex-specific differences in recombination originate at, or prior to, establishment of double-strand breaks. PLoS One 8:e85075.

49. Wang $S$, et al. (2017) Inefficient crossover maturation underlies elevated aneuploidy in human female meiosis. Cell 168:977-989.

50. Bojko M (1985) Human meiosis IX. Crossing over and chiasma formation in oocytes. Carlsberg Res Commun 50:43-72.

51. Brooks LD, Marks RW (1986) The organization of genetic variation for recombination in Drosophila melanogaster. Genetics 114:525-547.

52. Kong A, et al. (2002) A high-resolution recombination map of the human genome. Nat Genet 31:241-247.

53. Muralidhar $P$, Veller $C$ (2018) Sexual antagonism and the instability of environmental sex determination. Nat Ecol Evol 2:343-351.

54. Hartl DL, Ruvolo M (2012) Genetics: Analysis of Genes and Genomes (Jones \& Bartlett Learning, Burlington, MA), 8th Ed.

55. Darlington CD (1932) Recent Advances in Cytology (Churchill, London).

56. Darlington CD (1937) The biology of crossing-over. Nature 140:759-761.

57. Darlington CD (1939) The Evolution of Genetic Systems (Cambridge Univ Press, Cambridge, UK).

58. Zhao H, McPeek MS, Speed TP (1995) Statistical analysis of chromatid interference. Genetics 139:1057-1065.

59. Levan A (1935) Cytological studies in Allium, VI. The chromosome morphology of some diploid species of Allium. Hereditas 20:289-330.

60. Kauppi L, Jeffreys AJ, Keeney S (2004) Where the crossovers are: Recombination distributions in mammals. Nat Rev Genet 5:413-424.

61. Levine RP, Levine EE (1954) The genotypic control of crossing over in Drosophila pseudoobscura. Genetics 39:677-691.

62. Levine RP, Levine EE (1955) Variable crossing over arising in different strains of Drosophila pseudoobscura. Genetics 40:399-405.

63. Whitehouse C, Edgar L, Jones G, Parker J (1981) The population cytogenetics of Crepis capillaris I. Chiasma variation. Heredity 47:95-103.

64. Hinch AG, et al. (2011) The landscape of recombination in African Americans. Nature 476:170-175.

65. Wegmann $D$, et al. (2011) Recombination rates in admixed individuals identified by ancestry-based inference. Nat Genet 43:847-853.

66. Callan HG, Perry PE (1977) Recombination in male and female meiocytes contrasted. Philos Trans R Soc Lond B Biol Sci 277:227-233.

67. Fletcher HL, Hewitt GM (1980) A comparison of chiasma frequency and distribution between sexes in three species of grasshoppers. Chromosoma 77:129-144.

68. Young A (2014) The evolutionary feedback between genetic conflict and genome architecture. PhD thesis (Harvard University, Cambridge, MA).

69. Johnston SE, Huisman J, Ellis PA, Pemberton JM (2017) A high-density linkage map reveals sexually-dimorphic recombination landscapes in red deer (Cervus elaphus). G3 (Bethesda) 7:2859-2870.

70. King M, Hayman D (1978) Seasonal variation of chiasma frequency in Phyllodactylus marmoratus (Gray)(Gekkonidae - Reptilia). Chromosoma 69:131-154.

71. Laurie D, Jones G (1981) Inter-individual variation in chiasma distribution in Chorthippus brunneus (Orthoptera: Acrididae). Heredity 47:409-416.

72. Sun F, et al. (2006) Variation in MLH1 distribution in recombination maps for individual chromosomes from human males. Hum Mol Genet 15:2376-2391.

73. Cheung VG, Burdick JT, Hirschmann D, Morley M (2007) Polymorphic variation in human meiotic recombination. Am J Hum Genet 80:526-530.

74. Froenicke L, Anderson LK, Wienberg J, Ashley T (2002) Male mouse recombination maps for each autosome identified by chromosome painting. Am J Hum Genet 71:1353-1368.

75. Sun F, et al. (2004) Human male recombination maps for individual chromosomes. Am J Hum Genet 74:521-531.

76. White MJD (1973) Animal Cytology and Evolution (Cambridge Univ Press, Cambridge, UK), 3rd Ed.

77. Sherman PW (1979) Insect chromosome numbers and eusociality. Am Nat 113:925935.

78. Weissman DB (1976) Geographical variability in the pericentric inversion system of the grasshopper Trimerotropis pseudofasciata. Chromosoma 55:325-347.

79. Fletcher HL (1988) Sex and recombination. Nature 331:492.

80. Gorlov IP, Zhelezova Al, Gorlova OY (1994) Sex differences in chiasma distribution along two marked mouse chromosomes: Differences in chiasma distribution as a reason for sex differences in recombination frequency. Genet Res 64:161166.

81. Colombo PC (1992) A new index for estimating genetic recombination from chiasma distribution data. Heredity 69:412-415.

82. Haag CR, Theodosiou L, Zahab R, Lenormand T (2017) Low recombination rates in sexual species and sex-asex transitions. Philos Trans R Soc B Biol Sci 372:20160461.

83. Simpson EH (1949) Measurement of diversity. Nature 163:688-688.

84. Jost L (2006) Entropy and diversity. Oikos 113:363-375.

85. Crow JF (1988) The importance of recombination. The Evolution of Sex: An Examination of Current Ideas, eds Michod RE, Levin BR (Sinauer, Sunderland, MA), pp 56-73.

86. Burt A (2000) Perspective: Sex, recombination, and the efficacy of selection-was Weismann right? Evolution 54:337-351.

87. Cleland RE (1972) Oenothera: Cytogenetics and Evolution (Academic, London).

88. Sturtevant AH (1913) The linear arrangement of six sex-linked factors in Drosophila, as shown by their mode of association. J Exp Zoo A 14:43-59.

89. Muller HJ (1916) The mechanism of crossing-over. Am Nat 50:193-221. 
90. Anderson LK, Reeves A, Webb LM, Ashley T (1999) Distribution of crossing over on mouse synaptonemal complexes using immunofluorescent localization of MLH1 protein. Genetics 151:1569-1579.

91. Møens PB, et al. (2002) The time course and chromosomal localization of recombination-related proteins at meiosis in the mouse are compatible with models that can resolve the early DNA-DNA interactions without reciprocal recombination. $J$ Cell Sci 115:1611-1622.

92. Shifman S, et al. (2006) A high-resolution single nucleotide polymorphism genetic map of the mouse genome. PLOS Biol 4:e395.

93. Roach JC, et al. (2010) Analysis of genetic inheritance in a family quartet by wholegenome sequencing. Science 328:636-639.

94. Wang J, Fan HC, Behr B, Quake SR (2012) Genome-wide single-cell analysis of recombination activity and de novo mutation rates in human sperm. Cell 150:402-412.

95. Lu S, et al. (2012) Probing meiotic recombination and aneuploidy of single sperm cells by whole-genome sequencing. Science 338:1627-1630.

96. Mancera E, Bourgon R, Brozzi A, Huber W, Steinmetz LM (2008) High-resolution mapping of meiotic crossovers and noncrossovers in yeast. Nature 454:479-485.

97. Hou Y, et al. (2013) Genome analyses of single human oocytes. Cell 155:1492-1506.

98. Cole $F$, et al. (2014) Mouse tetrad analysis provides insights into recombination mechanisms and hotspot evolutionary dynamics. Nat Genet 46:1072-1080.

99. von Wettstein D, Rasmussen SW, Holm PB (1984) The synaptonemal complex in genetic segregation. Annu Rev Genet 18:331-411.

100. Sherman JD, Stack SM (1995) Two-dimensional spreads of synaptonemal complexes from solanaceous plants. VI. High-resolution recombination nodule map for tomato (Lycopersicon esculentum). Genetics 141:683-708.

101. Zickler D, Kleckner N (1999) Meiotic chromosomes: Integrating structure and function. Annu Rev Genet 33:603-754.

102. Anderson LK, et al. (2014) Combined fluorescent and electron microscopic imaging unveils the specific properties of two classes of meiotic crossovers. Proc Natl Acad SCi USA 111:13415-13420.

103. Hassold T, Sherman S, Hunt $P(2000)$ Counting cross-overs: Characterizing meiotic recombination in mammals. Hum Mol Genet 9:2409-2419.

104. Hunter N (2015) Meiotic recombination: The essence of heredity. Cold Spring Harbor Perspect Biol 7:a016618.

105. Fransz P, et al. (1998) Cytogenetics for the model system Arabidopsis thaliana. Plant J 13:867-876.

106. Ruiz-Herrera A, et al. (2017) Recombination correlates with synaptonemal complex length and chromatin loop size in bovids-insights into mammalian meiotic chromosomal organization. Chromosoma 126:615-631.

107. Møens PB, Pearlman RE (1988) Chromatin organization at meiosis. Bioessays 9:151153.

108. Kleckner N (2006) Chiasma formation: Chromatin/axis interplay and the role (s) of the synaptonemal complex. Chromosoma 115:175-194.

109. Li S, Meistrich M, Brock W, Hsu T, Kuo M (1983) Isolation and preliminary characterization of the synaptonemal complex from rat pachytene spermatocytes. Exp Cell Res 144:63-72.

110. Kleckner N, Storlazzi A, Zickler D (2003) Coordinate variation in meiotic pachytene SC length and total crossover/chiasma frequency under conditions of constant DNA length. Trends Genet 19:623-628.

111. Novak I, et al. (2008) Cohesin $\mathrm{Smc1} \beta$ determines meiotic chromatin axis loop organization. J Cell Biol 180:83-90.

112. Keyl HG (1975) Lampbrush chromosomes in spermatocytes of Chironomus. Chromosoma 51:75-91.

113. Pukkila PJ, Lu BC (1985) Silver staining of meiotic chromosomes in the fungus, Coprinus cinereus. Chromosoma 91:108-112.

114. Lu BC (1993) Spreading the synaptonemal complex of Neurospora crassa. Chromosoma 102:464-472.

115. Zhang L, Liang Z, Hutchinson J, Kleckner N (2014) Crossover patterning by the beam film model: Analysis and implications. PLoS Genet 10:e1004042.

116. Handyside $\mathrm{AH}$, et al. (2010) Karyomapping: A universal method for genome wide analysis of genetic disease based on mapping crossovers between parental haplotypes. J Med Genet 47:651-658.

117. Coop G, Wen X, Ober C, Pritchard JK, Przeworski M (2008) High-resolution mapping of crossovers reveals extensive variation in fine-scale recombination patterns among humans. Science 319:1395-1398.

118. Chowdhury R, Bois PRJ, Feingold E, Sherman SL, Cheung VG (2009) Genetic analysis of variation in human meiotic recombination. PLoS Genet 5:e1000648.

119. Fan HC, Wang J, Potanina A, Quake SR (2011) Whole-genome molecular haplotyping of single cells. Nat Biotech 29:51-57.

120. Jeffreys AJ, et al. (2004) Meiotic recombination hot spots and human dna diversity. Philos Trans R Soc B 359:141-152.

121. Kosambi DD (1944) The estimation of map distance from recombination values. Ann Eugen 12:172-175

122. Lian J, et al. (2008) Variation in crossover interference levels on individual chromosomes from human males. Hum Mol Genet 17:2583-2594.

123. Kong $\mathrm{A}$, et al. (2008) Sequence variants in the RNF212 gene associate with genomewide recombination rate. Science 319:1398-1401.
124. Ghiselin MT (1974) The Economy of Nature and the Evolution of Sex (University of California Press, Berkeley, CA).

125. Maynard Smith J (1976) A short-term advantage for sex and recombination through sib-competition. J Theor Biol 63:245-258.

126. Goldstein DB, Bergman A, Feldman MW (1993) The evolution of interference: Reduction of recombination among three loci. Theor Pop Biol 44:246-259.

127. Gorlov IP, Gorlova OY (2001) Cost-benefit analysis of recombination and its application for understanding of chiasma interference. J Theor Biol 213:1-8.

128. Hillers KJ (2004) Crossover interference. Curr Biol 14:R1036-R1037.

129. Wang S, Zickler D, Kleckner N, Zhang L (2015) Meiotic crossover patterns: Obligatory crossover, interference and homeostasis in a single process. Cell Cycle 14:305-314.

130. Lenormand T, Engelstädter J, Johnston SE, Wijnker E, Haag CR (2016) Evolutionary mysteries in meiosis. Philos Trans R Soc B Biol Sci 371:20160001.

131. Saito TT, Colaiácovo MP (2017) Regulation of crossover frequency and distribution during meiotic recombination. Cold Spring Harb Symp Quant Biol 82:223-234.

132. van Veen J, Hawley R (2003) Meiosis: When even two is a crowd. Curr Biol 13:R831R833.

133. Koszul R, Meselson M, Van Doninck K, Vandenhaute J, Zickler D (2012) The centenary of Janssens's chiasmatype theory. Genetics 191:309-317.

134. Egel R (1995) The synaptonemal complex and the distribution of meiotic recombination events. Trends Genet 11:206-208.

135. Fernandes JB, Seguéla-Arnaud $M$, Larchevêque $C$, Lloyd AH, Mercier $R$ (2018) Unleashing meiotic crossovers in hybrid plants. Proc Natl Acad Sci USA 115:2431-2436.

136. Serra $\mathrm{H}$, et al. (2018) Massive crossover elevation via combination of $H E / 10$ and recq4a recq4b during Arabidopsis meiosis. Proc Natl Acad Sci USA 115:2437-2442.

137. Crow JF, Kimura M (1965) Evolution in sexual and asexual populations. Am Nat 99:439-450.

138. Weinreich DM, Chao L (2005) Rapid evolutionary escape by large populations from local fitness peaks is likely in nature. Evolution 59:1175-1182.

139. Neher RA, Shraiman BI (2009) Competition between recombination and epistasis can cause a transition from allele to genotype selection. Proc Natl Acad Sci USA 106:68666871

140. Hartl DL (1975) Modifier theory and meiotic drive. Theor Popul Biol 7:168-174

141. Liberman U (1976) Modifier theory of meiotic drive: Is Mendelian segregation stable? Theor Popul Biol 10:127-132.

142. Charlesworth B, Hartl DL (1978) Population dynamics of the segregation distorte polymorphism of Drosophila melanogaster. Genetics 89:171-192.

143. Rice WR (1986) On the instability of polygenic sex determination: The effect of sex specific selection. Evolution 40:633-639.

144. van Doorn GS, Kirkpatrick M (2007) Turnover of sex chromosomes induced by sexua conflict. Nature 449:909-912.

145. van Doorn GS, Kirkpatrick M (2010) Transitions between male and female heterogamety caused by sex-antagonistic selection. Genetics 186:629-645

146. McVean GAT, Charlesworth B (2000) The effects of Hill-Robertson interference between weakly selected mutations on patterns of molecular evolution and variation. Genetics 155:929-944.

147. Beliveau BJ, et al. (2012) Versatile design and synthesis platform for visualizing genomes with Oligopaint FISH probes. Proc Natl Acad Sci USA 109:21301-21306.

148. Beliveau BJ, et al. (2015) Single-molecule super-resolution imaging of chromosomes and in situ haplotype visualization using Oligopaint FISH probes. Nat Comm 6 : 7147.

149. Wang S, et al. (2016) Spatial organization of chromatin domains and compartments in single chromosomes. Science 353:598-602.

150. Eddy SR (2012) The C-value paradox, junk DNA and ENCODE. Curr Biol 22:R898-R899.

151. Eddy SR (2013) The ENCODE project: Missteps overshadowing a success. Curr BiO 23:R259-R261.

152. Lek M, et al. (2016) Analysis of protein-coding genetic variation in 60,706 humans Nature 536:285-291.

153. The 1000 Genomes Project Consortium (2015) A global reference for human genetic variation. Nature 526:68-74.

154. Spencer CC, et al. (2006) The influence of recombination on human genetic diversity. PLoS Genet 2:e148.

155. Cole F, Keeney S, Jasin M (2012) Preaching about the converted: How meiotic gene conversion influences genomic diversity. Ann NY Acad Sci 1267:95-102.

156. Zelazowski MJ, et al. (2017) Age-dependent alterations in meiotic recombination cause chromosome segregation errors in spermatocytes. Cell 171:601-614.

157. Kleckner N, Zhang L, Weiner B, Zickler D (2012) Meiotic chromosome dynamics. Genome Organization and Function in the Cell Nucleus, ed Rippe K (Wiley-VCH Weinheim, Germany), pp 487-533.

158. Cole F, et al. (2012) Homeostatic control of recombination is implemented progressively in mouse meiosis. Nat Cell Biol 14:424-430.

159. Andolfatto $P$, Nordborg M (1998) The effect of gene conversion on intralocus associations. Genetics 148:1397-1399.

160. Lee PS, et al. (2009) A fine-structure map of spontaneous mitotic crossovers in the yeast Saccharomyces cerevisiae. PLoS Genet 5:e1000410.

161. Hum YF, Jinks-Robertson S (2017) Mitotic gene conversion tracts associated with repair of a defined double-strand break in Saccharomyces cerevisiae. Genetics 207:115-128. 